\title{
Role of the Basal Ganglia in Balance Control
}

\author{
Jasper E. Visser and Bastiaan R. Bloem \\ Department of Neurology, University Medical Center St Radboud, Nijmegen, the Netherlands
}

\begin{abstract}
SUMMARY
In this review paper, we summarize the important contributions of the basal ganglia to the regulation of postural control. After a brief overview of basal ganglia circuitries, the emphasis is on clinical observations in patients with focal lesions in parts of the basal ganglia, as the impairments seen here can serve to highlight the normal functions of the basal ganglia nuclei in postural control. Two particularly relevant functions are discussed in detail: first, the contribution of the basal ganglia to flexibility and to gaining control of balancecorrecting responses, including the ability to lend priority to the elements of a postural task; and second, processing afferent information by the basal ganglia, which is increasingly recognized as being highly relevant for postural control.
\end{abstract}

\section{INTRODUCTION}

The term basal ganglia refers to a group of subcortical nuclei that includes the striatum, globus pallidus, and connected structures. The striatum is divided into a dorsal division that includes the caudate and putamen, and a ventral division that

Reprint requests to: Jasper E. Visser, MD, University Medical Center St Radboud, Department of Neurology (326), P.O. Box 9101, $6500 \mathrm{HB}$ Nijmegen, the Netherlands; Email: j.visser@neuro.umcn.nl includes the amygdala and olfactory tubercle. The subthalamic nucleus and the substantia nigra (SN) are often considered together with the basal ganglia because of their important reciprocal connections.

Functionally, the basal ganglia have long been regarded to be predominantly involved in motor control but are now increasingly recognized to play an additional role in sensory processing, cognition, and behavior (Brown et al., 1997; Bloem et al., 2001d; Bhatia \& Marsden, 1994). Major functions of the basal ganglia and examples of the consequences of basal ganglia dysfunction for postural control are shown in Table 1 .

Here we will focus on the important and versatile role of the basal ganglia in controlling balance. Our aim is to provide an overview of the role of the basal ganglia in balance control by reviewing observations on postural control in patients with basal ganglia lesions. The first section will briefly describe the neuroanatomy and neurochemistry of basal ganglia circuits and their connections. In particular, the basal ganglia connections with brainstem nuclei will be discussed as these are increasingly recognized as contributing to postural control.

Next, the role of several basal ganglia nuclei in postural control will be illustrated by clinical observations on patients with focal basal ganglia lesions as this underscores the balance-related functions of the basal ganglia.

Finally, we will discuss several aspects of balance control in which the basal ganglia might be involved, including motor programming, muscle tone control, and sensory-motor integration. Indirectly, the observations on elderly patients can 
TABLE 1

Functions of the basal ganglia that might be relevant for postural control

\section{Basal ganglia function}

\begin{tabular}{ll}
\hline - Storing and automatic execution of motor plans & - Gait akinesia / freezing \\
$\begin{array}{l}\text { - Motor flexibility, adaptive behavior to } \\
\text { environmental changes }\end{array}$ & - Postural "inflexibility" \\
- Somatosensory integration & - Stooped posture \\
& - Contraversive pushing \\
- Muscle tone regulation & - Axial stiffness \\
- Gain control of automatic postural responses & - Exaggerated destabilizing responses \\
& - Diminished stabilizing responses \\
- Cognition, motivation and emotional aspects of & - Co-contraction \\
behavior & - Impaired scaling of postural responses under \\
& conditions of uncertainty \\
\end{tabular}

shed some light on the developmental aspects of the basal ganglia and balance, a field that has otherwise received little scientific attention. Indeed, brain lesions in patients with degenerative diseases can provide insight into the possible role of the basal ganglia in the pathophysiology of developmental disorders.

\section{ORGANIZATION OF THE BASAL GANGLIA AND PATHWAYS}

The neural connections within the basal ganglia and their projections are complicated (Parent \& Hazrati, 1995a; Hamani et al., 2004; Pahapill \& Lozano, 2000). Several simplified models have been proposed. The traditional model recognizes several parallel loops through the basal ganglia (Alexander \& Crutcher, 1990). These loops share a common neuroanatomical plan, but each serves specific motor, cognitive, or behavioral functions in a more or less segregated manner. Although these models are oversimplified and not true reflections of the actual anatomical situation (Chesselet \& Delfs, 1996; Parent \& Cicchetti, 1998), they do have important value as conceptual models for discussing the functional consequences of localized lesions.

A schematic representation of a basal ganglia circuit and the major neurotransmitters involved is represented in Fig. 1. Cortical input is directed to the striatum, via glutamatergic projections (Albin et al., 1995; Parent \& Hazrati, 1995b). At the striatal level, the putamen seems mostly involved in motor tasks, whereas cognitive and behavioral functions are served by the caudate nucleus and the ventral striatum. The dorsal striatum projects to the globus pallidus pars interna $(\mathrm{GPi})$ and to the 


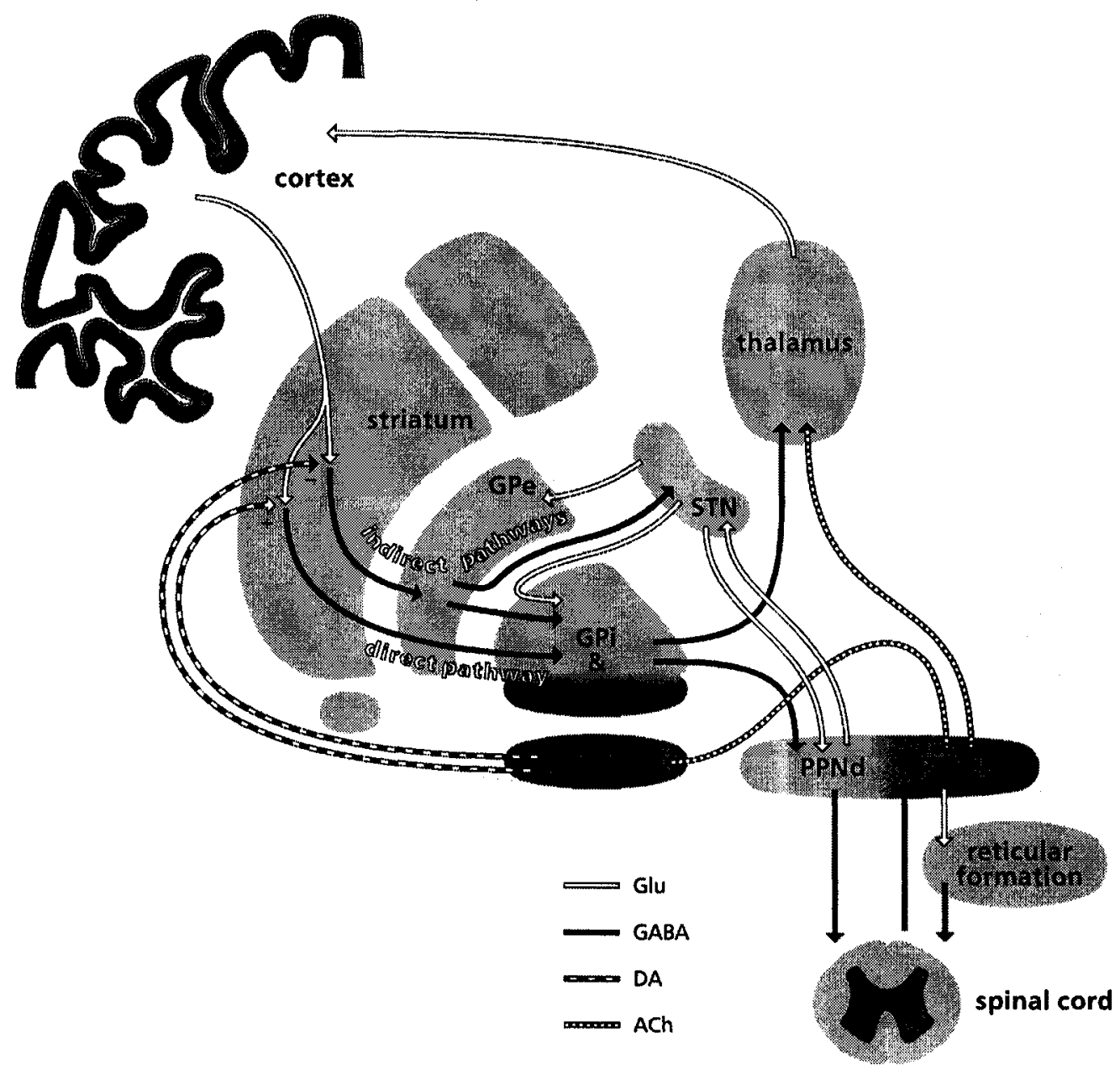

Fig. 1. Schematic model of the basal ganglia. Abbreviations: ACh, acetylcholine; DA, dopamine; GABA, gammahydroxy butyric acid; Glu, glutamate; GPe, globus pallidus, pars externa; GPi, globus pallidus, pars interna; PPNc, pedunculopontine nucleus, pars compacta; PPNd, pedunculopontine nucleus, pars dissipatus; SNc, substantia nigra, pars compacta; SNr, substantia nigra, pars reticulata; STN, nucleus subthalamicus. Note that the projections of these basal ganglia circuitries are not only "ascending" to the motor cortex, but also "descending" to brainstem nuclei, the reticular formation and the spinal cord. These descending connections are increasingly recognized as important for various aspects of postural control. 
pars reticulata of the substantia nigra $(\mathrm{SNr})$, two areas that share many functional and structural properties, often jointly regarded as the "output nuclei" of the basal ganglia (Parent \& Hazrati, 1995a,b). Two separate pathways within these striatopallidal projections are recognized: a direct pathway projecting from the striatum to the $\mathrm{GPi} / \mathrm{SNr}$, and an indirect pathway that connects the striatum to the $\mathrm{Gpi} / \mathrm{SNr}$ via the $\mathrm{GPe}$ and the subthalamic nucleus (STN). Based on the excitatory and inhibitory nature of the connections, the direct and indirect pathways through the basal ganglia have opposing effects on net basal ganglia output. The activation of direct pathways tends to decrease inhibitory GPi/SNr output, whereas striatal excitation increases inhibitory output via indirect pathways. Finally, the GPi/SNr connects to specific nuclei in the thalamus, and thalamic efferents project back to cortical areas.

In addition to the input from cortical areas, the striatum receives dopaminergic input from the pars compacta of the substantia nigra $(\mathrm{SNc}$ ) (Albin et al., 1995; Parent \& Hazrati, 1995a,b; Alexander \& Crutcher, 1990). Dopamine probably has opposing effects on the direct and indirect pathways, such that the overall impact of dopamine is facilitative in nature by decreasing inhibitory basal ganglia output.

In addition to the thalamo-cortical projections, basal ganglia output is also directed to the brainstem and spinal cord (Fig. 1). Specifically, GPi and SNr neurons projecting to the thalamus also send collaterals to the pedunculopontine nucleus (PPN) and other midbrain areas, including the superior colliculus (Parent \& Hazrati, 1995a,b). The main inputs to the PPN stem not only from the globus pallidus and $\mathrm{SNr}$ but also from the STN and spinal cord (Pahapill \& Lozano, 2000). The ascending PPN output connects to all parts of the basal ganglia. The descending PPN output projects to the medullary reticular formation, relaying bilaterally to the spinal cord. Direct projections from the PPN to the spinal cord have also been described.

The anatomical and physiological properties of the PPN have been reviewed recently (Pahapill \& Lozano, 2000). The PNN consists of a heterogeneous group of neurons, located in the upper brainstem. The PPN can be regarded as part of the midbrain locomotor region (MLR), a functionally defined area involved in controlling locomotion. Two main neuronal populations exist within the PPN: the PPN compacta (PPNc), consisting of mainly cholinergic neurons; and the PPN dissipatus (PPNd), consisting of both cholinergic and glutamatergic neurons. Dysfunctional projections via the PPN may account for certain phenomena in basal ganglia disease-including gait and balance impairment-that cannot be explained by traditional basal ganglia models (Lee et al., 2000; Pahapill \& Lozano, 2000).

\section{POSTURAL CONSEQUENCES OF BASAL GANGLIA LESIONS}

Why are the basal ganglia thought to contribute to balance control? One line of evidence stems from clinical neurology, in which many concepts of structure-function relationships are based on classical lesion studies in patients and animals with focal lesions. That is, the function of a certain structure is suggested by the functional loss after focal damage to that specific structure. In this section, we will explore the consequences of focal brain lesions for postural control and balance. Balance impairment has frequently been observed in patients with basal ganglia lesions, e.g. in patients with degenerative, vascular, post-infectious, toxic, neoplastic, or iatrogenic lesions of the basal ganglia (Bloem \& Bhatia, 2004). In this section, the reported effects of focal lesions of different areas in the basal ganglia and several of their target areas on balance control will be discussed. 


\subsection{Lesions in the basal ganglia}

The best-known human example of a lesion in the substantia nigra (SN) is Parkinson's disease (PD), a disorder predominantly characterized by degeneration of dopaminergic cells in the $\mathrm{SNr}$ (Samii et al., 2004). In PD, falling is frequent, as up to 70 percent of patients falls at least once each year, and some 50 percent of subjects fall twice or more each year (Bloem et al., 2004a). The balance disorder in PD can be regarded part of a spectrum of motor dysfunctions in axial muscles, termed "axial apraxia" (Lakke, 1985). Other examples include the difficulty in turning around the vertical axis and the characteristically stooped posture. The presence of these abnormalities in PD suggests an involvement of the $\mathrm{SNr}$ and its dopaminergic projections in normal axial motor control. Note that lesions in PD patients extend beyond the SN, certainly in more advanced disease stages. Other non-dopaminergic areas like the locus coeruleus can then become involved and are likely to contribute to balance impairment (Bloem et al., 2001c).

In humans, acquired and more selective $\mathrm{SN}$ lesions have been studied in persons exposed to a neurotoxic heroin analogue, 1-methyl-4-phenyl1,2,3,6-tetrahydropyridine (MPTP), that rather selectively destroys nigrostratiatal neurons in the $\mathrm{SN}$. This destruction is associated with a marked reduction of central dopaminergic function in the putamen and caudate (Snow et al., 2000). The symptoms in MPTP patients are strikingly similar to those in PD patients, including gait disorders, balance impairment, and falling (Bloem \& Roos, 1995). The association of a selective hypodopaminergic state with axial mobility deficits suggests a role for the $\mathrm{SN}$ in at least certain elements of gait and balance control.

Published reports on the effects of selective putaminal lesions on postural control are scarce. One report of nine patients with sudden falls due to vascular basal ganglia lesions described an involvement of the putamen in seven subjects (Labadie et al., 1989). These patients fell "like a log", apparently not aware of the fall and hardly using protective movements to prevent falling. Such observations suggest that the putamen somehow contributes to balance control, although the exact function remains unclear. As stated before, caudate lesions rarely cause motor disorders but rather are associated with cognitive and behavioral problems, including apathy and disinhibition (Bhatia \& Marsden, 1994; Alexander \& Crutcher, 1990).

Important observations were made in PD patients who had undergone stereotactic deep-brain surgery of the basal ganglia as a treatment. Interventions targeted at the GPi included stereotactic lesions or high-frequency electrical stimulation achieving the same net effect as a lesion. Unilateral interventions can reduce contralateral symptoms and can lead to a mild, clinically noticeable improvement of posture and gait impairment (Bakker et al., 2004). Bilateral interventions can be associated with disabling side-effects, including marked postural instability. Such contrasting clinical effects on postural control-beneficial on the one hand, deleterious on the other-suggest an involvement of the GPi in balance control.

An involvement of the STN in postural control has been suggested by the outcomes of PD patients subjected to deep brain surgery. Various studies reported improvement following bilateral STN stimulation (see e.g. Limousin et al., 1998; DeepBrain Stimulation for Parkinson's Disease Study Group, 2001; Bejjani et al., 2000; Krack et al., 2003). Adverse effects can also occur, however, as postural instability can deteriorate in certain patients (Bakker et al., 2004). Two patients who underwent a unilateral subthalamotomy showed a marked postoperative postural asymmetry - a lateral curvature of the spine with head and body tilting toward the side contralateral to the STN lesion (Su et al., 2002). Although apparently aware of their instability, 
these patients seemed to neglect their body drift. Dopaminergic medication caused partial improvement, but contralateral surgery corrected the abnormality. These observations underscore the involvement of the STN in balance control.

\subsection{Lesions in efferent connections}

Additional indirect evidence comes from observations on patients with balance impairment caused by focal lesions in areas that receive abundant projections from the basal ganglia. Two important structures include the thalamus and the PPN, both possibly serving as final pathways via which the basal ganglia influence balance and postural control.

The thalamus seems involved in axial motor control in multiple ways. First, the thalamus may function as a relay structure for the sensory input of different modalities to the cortex, and could as such be involved in the graviceptive system. For example, patients with acute thalamic infarcts can show a tilt of the subjective visual vertical (Karnath et al., 2000). Additional evidence suggesting an involvement of the thalamus in the graviceptive system has been suggested by observing certain stroke patients, who not only show instability due to hemiplegia but also actively push toward the hemiplegic side. In such patients, the posterolateral thalamus is predominantly involved (Karnath et al., 2000). This involvement apparently alters the perception of the body's orientation in relation to gravity, leading to the perception of being upright while the body is actually tilted. The importance of the thalamus for postural control is further underscored by the effects of deep-brain surgery targeted at the thalamus. Unilateral approaches may improve postural control, perhaps by decreasing tremor, but bilateral interventions often cause severe balance impairment (Speelman, 1991).

Degeneration of the PPN occurs in various neurodegenerative disorders, including PD, and can be particularly prominent in progressive supranuclear palsy (PSP) (Bloem \& Bhatia, 2004). In patients with this disorder, balance impairment is often pronounced, and falling is even more frequent than in PD, occurring already in early disease stages (Bloem et al., 2004b). The severity of balance impairment in PSP is perhaps related to a marked cell loss within the PPN (Hirsch et al., 1987). Reports on patients with selective PPN lesions are rare. One patient with a hemorrhagic lesion involving the right PPN reportedly could not stand or walk (Masdeu et al., 1994). Focal lesions in the PPN have been performed in primates, however, in which bilateral lesions resulted in permanent akinesia (Munro-Davies et al., 1999). Furthermore, the electrical stimulation of the PPN in macaques was associated with frequencydependent effects. Stimulation at $100 \mathrm{~Hz}$ caused impairment of postural control and rendered the monkey unable to balance itself (Nandi et al., 2002b). Furthermore, in monkeys with MPTPparkinsonism, injection of the GABA antagonist bicuculline into the PPN resulted in significant symptom reduction (Nandi et al., 2002a). The results of these animal experiments underline the important role of the PPN in balance control.

\section{BASAL GANGLIA AND SPECIFIC BALANCE FUNCTIONS}

We will next explore two essential functional domains of postural control-the regulation of postural flexibility and the control of sensorimotor integration-that might be governed, at least in part, by the basal ganglia. The emphasis will be on PD, for which most observations have been made.

\subsection{Postural in flexibility}

The basal ganglia are increasingly recognized as critical structures involved in flexibility or set- 
shifting, including mental flexibility as well as motor flexibility. Being flexible is an indispensable feature of all living organisms because flexibility allows them to adapt to a changing environment. Balancing also requires continuous adaptation to a rapidly changing environment, being caused either by externally generated perturbations, such as a moving support surface, or by self-initiated movements like rising from a chair.

To cope with sudden balance perturbations, several mechanisms exist. The first are purely passive mechanisms, such as the body's inertia. Second, "automatic" responses are defined as balance-correcting responses, whose onset latency is too early for a voluntary response but strikingly flexible in magnitude, depending on the specific task at hand. Finally, voluntary balance corrections are those that are purposefully initiated. The importance of the basal ganglia in fine-tuning all these mechanisms has been demonstrated in posturography experiments, the quantitative assessment of human upright stance during quiet stance or in response to standardized bodily perturbations (Bloem et al., 2003).

Various investigators observed that postural control in PD is characterized by stiffening and reduced flexibility. For example, evidence has been obtained for axial stiffness in PD patients who were exposed to relatively slow platform tilts in the sagittal plane (Maurer et al., 2003). In addition, PD patients who are exposed to more rapid displacements of an underlying movable platform also show signs of stiffness at the ankles (Bloem et al., 1996) and at the pelvis and trunk (Carpenter et al., 2004). Such postural inflexibility is associated with frequent loss of balance on the platform, presumably because stiffness reduces the body's ability to dampen the impact of the external perturbation passively, by hinging at the hips. We should note that stiffening in itself does not invariably have a deleterious effect on balance control because it can help to reduce the number of degrees of freedom that have to be controlled.

Several mechanisms can explain the stiffness in PD patients. First, joint motion can be constrained because of increased muscle stiffness, which indeed occurs in ankle muscles of PD patients (Dietz et al., 1988). The muscle stiffness could be caused by secondary changes in intrinsic muscle properties.

Second, stiffening could result from tonic increases in background muscle activity, even before postural perturbations have occurred. Such tonically increased background activity has indeed been observed in PD (Schieppati \& Nardone, 1991; Horak et al., 1996). These findings suggest that the basal ganglia, in particular the $\mathrm{SNr}$ and its projections to the upper dorsal brainstem, help to optimize muscle tone for the ongoing gait or balance task (Takakusaki et al., 2004).

Finally, stiffening could be related to an impaired scaling of automatic postural responses. We will illustrate this for a common experimental set-up, using toe-up rotations of a supporting platform upon which subjects are standing. Such perturbations move the subject backward and elicit a monosynaptic spinal stretch response and a socalled medium latency (ML) response in the stretched triceps surae. These responses are followed by a long-latency (LL) postural response in the shortened tibialis anterior. The ML activity has a destabilizing effect, whereas the LL responses in the shortened antagonist help to stabilize posture. When PD patients are confronted with identical toe-up rotational perturbations, several differences emerge (Allum et al., 1988; Bloem et al., 1996). First, PD patients have enlarged ML stretch responses, and this enlargement of a destabilizing response is thought to induce postural instability. Second, patients have LL responses that are smaller than normal, and a reduction of a normally stabilizing response might further lead to postural instability. Indeed, PD patients are more unstable than controls on the moving platform, as reflected 
by a larger posterior sway (Bloem et al., 1996). This instability suggests that $\mathrm{PD}$ patients have a fundamental problem in scaling the magnitude of their postural responses. Later studies extended these observations by using platforms that could suddenly rotate or translate in multiple directions (multidirectional dynamic posturography) (Carpenter et al., 2004; Dimitrova et al., 2004). A key finding in these studies was a directional preponderance for falls in a backward direction. This backward falling was again related to an abnormal scaling of balance correcting responses, causing co-contraction between agonist and antagonist muscles. Cocontraction was also a common finding in other posturography studies of PD patients (Dietz et al., 1995; Horak et al., 1992). It remains unclear, however, whether co-contraction is purely an expression of primary dysfunction or at least partly a compensatory strategy to cause stiffness (and fewer degrees of freedom) when other mechanisms fail.
The central nervous system can normally adapt the postural strategies to meet the functional demands of the ongoing postural task (postural set). The term postural set covers a wide array of conditions, including changes in the environmental situation, changes in the subject's initial body position and changes in the subject's perceptions about the postural task. Again, the basal ganglia seem to be an important mediator here, as PD patients have particular difficulties when postural set is manipulated.

This view can again be illustrated using the simple toe-up rotational paradigm. Under predictable conditions, small postural perturbations elicit comparably small stabilizing LL responses in healthy subjects, whereas larger perturbations elicit larger responses (Fig. 2a, 2b). When receiving a random mixture of small and large perturbations, young healthy subjects select a default postural response that is sufficiently large to cope with the largest possible perturbation (Beckley et al., 1991).
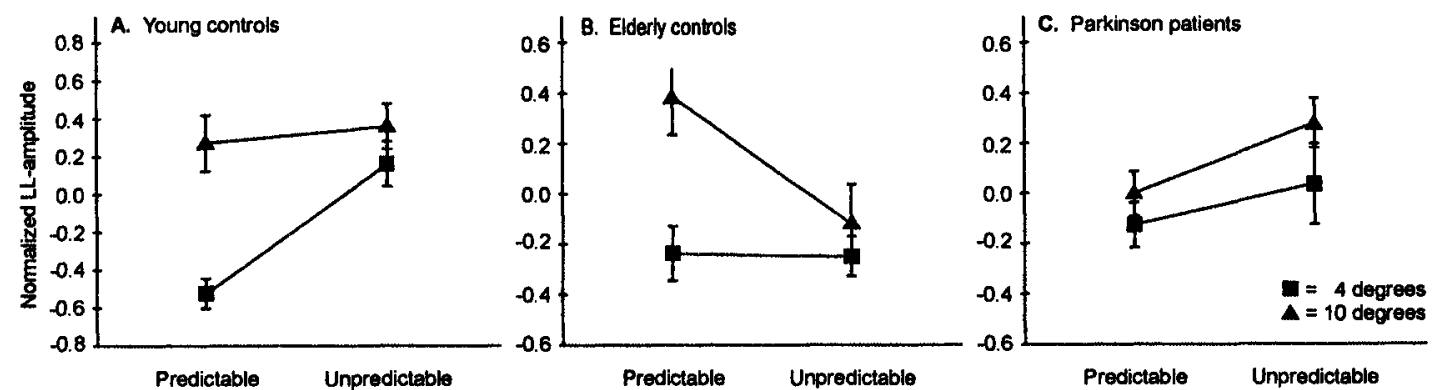

Fig. 2. Response amplitude scaling of tibialis anterior LL responses for 10 young healthy subjects (A), 13 elderly healthy subjects (B) and 12 patients with PD (C). All subjects were exposed to sudden toe-up rotational movements of supporting forceplate, the size of which could be varied between 4 degrees or 10 degrees amplitude either predictably (serial presentation of identically sized stimuli) or unpredictably (random mix of small and large perturbations). The graphs show the grand mean and SEM for normalized LL response amplitudes for each of the two levels of both independent variables: forceplate amplitude (4 degrees and 10 degrees) and degree of predictability ("predictable" or "unpredictable"). Data in this figure were modified from Beckley et al. (Beckley et al., 1993; Beckley et al., 1991) and reproduced from Bloem \& Bhatia (2004), with permission of the authors and the publisher. 
Older subjects also select such a 'default' response, albeit one that matches the smaller perturbation size. Thus, healthy subjects can modify the strength of their postural responses according to the demands of the task at hand. Figure 2c shows that this ability is lost in PD patients; they cannot modify the size of postural responses even though the magnitude of the perturbation is known in advance (Beckley et al., 1993). Furthermore, PD patients are unable to select a default response during unpredictable conditions, leading to a fixed response size under all conditions. Similar signs of 'inflexibility' have been observed under many different conditions (Horak et al., 1992; Schieppati \& Nardone, 1991; Bloem et al., 1995). The inability to modulate the size of postural responses properly is another reflection of abnormal gain control by the basal ganglia.

Not only shifting between different motor plans is important but also the ability to perform simultaneous tasks and-if necessary-arrange movements according to priority. The "stops walking when talking" test addresses this issue in the light of balance control (Lundin-Olsson et al., 1997). In this paradigm, subjects who are unable to maintain a routine conversation while walking have an increased risk of falls in the near future. Such an inability to execute two tasks simultaneously (one of them being a postural or gait task) can reflect a limited central processing capacity of the central nervous system. Patients with PD should be particularly vulnerable under such circumstances because the basal ganglia play an important role in the running of sequiential or simultaneous motor programs (Marsden, 1982). Indeed, in daily life many falls in PD patients occur under dual task circumstances (Willemsen et al., 2000). Surprisingly, when the "stops walking when talking" is delivered test to PD patients, this causes no problems for most patients (Bloem et al., 2000). Under much more challenging conditions in a truly multiple task design (Bloem et al., 2001b), however, including walking several meters and executing an increasing number of additional cognitive and motor tasks at the same time, PD patients perform poorer than do controls (Bloem et al., 2001a), as reflected by slowed or blocked performance. Patients were less able than agematched elderly controls to employ a "posture first" strategy but instead attempted to perform all tasks simultaneously. Nevertheless, because of the patients' balance impairment and restricted processing resources neither motor nor cognitive components were executed very successfully, resulting in a high proportion of errors. This finding might be interpreted as a form of 'risky' behavior that might lead to falls in daily life. Apparently, the basal ganglia are also involved in regulating such priority processes, perhaps by their ability to promote a rapid switch between different tasks.

\subsection{Sensorimotor integration}

The important role played by the basal ganglia in integrating and weighting afferent sensory information is increasingly becoming clear. This view is true for incoming feedback from the visual system, for the proprioceptive system, and for the vestibular system. Consequently, several motor deficits in patients with basal ganglia dysfunction should perhaps be viewed as-at least partiallydue to afferent disturbances.

We will briefly focus on studies that showed proprioceptive disturbances in PD. For example, the discrimination of differences in static positions of both elbow joints is impaired in PD (Zia et al., 2000). Furthermore, PD patients cannot actively match, with eyes closed, the position or trajectory of one arm to the position of the other arm, after this has been displaced actively by the patient (Klockgether et al., 1995; Zia et al., 2000). Other studies showed that PD patients find it difficult to 
accurately point to remembered visual targets in the dark, i.e. when proprioceptive information normally provides the most reliable source of information about finger position (Adamovich et al., 2001; Keijsers et al., 2005).

Information about the position of the actively moving arm normally originates from sensory kinesthetic feedback arising in the periphery during the actual movement, as well as from corollary discharges that contain information about movement plans from brain structures concerned with movement programming and execution. Therefore, problems in PD might arise from defective peripheral kinesthetic feedback because the afferent information itself is flawed, the information is abnormally processed at a central level, or because patients have abnormal corollary discharges. Studies in which arms are passively moved by the experimenter, therefore lacking corollary discharges, suggest that disturbed kinesthesia, rather than incorrect efference copies, causes hypometria in PD. For example, passive arm movements seem as hypometric as selfgenerated movements (Klockgether et al., 1995).

Central processing abnormalities within the basal ganglia could also affect postural control in various ways. For example, abnormal proprioceptive processing could explain the fixed gain of postural responses that underlies the postural inflexibility in PD. Another possibility is that patients have an abnormally constructed internal map of their stability limits and have lost their normal sense of limb and trunk position. Patients might also falsely perceive their subjective vertical to be shifted backward, forcing them to adopt a stooped posture (Kitamura et al., 1993). In addition, due to an uncertainty about actual body orientation in space, patients might assume a 'worst-case scenario' of constant instability, which causes a fear of falls. Both this fear and the actual impaired afferent input could cause stiffening, with adverse effects on dynamic postural control. In this light, it might be possible that impaired sensory processing is at least partly responsible for the stiffness and inflexibility as described in Sec. 4.1.

Although not exclusively seen in patients with basal ganglia lesions, some evidence supports the concept of incorrect self-perception resulting from abnormal basal ganglia function. We already mentioned the occasional occurrence of a seeming 'neglect' for lateral instability in patients with a unilateral lesion in, for example, the putamen or the subthalamic nucleus (Su et al., 2002; Labadie et al., 1989). In one experiment, patients were confronted with line drawings showing varying degrees of stoop and lateral deviation (Moore et al., 2000). When asked to identify the drawing that best resembles their self-perceived posture, patients were likely to underestimate the severity of their abnormalities. This finding suggests that patients have lost their normal sense of trunk position in space and supplements clinical experience that many patients are surprised to observe their own stooped posture in a mirror. Abnormal trunk position sense could also explain the sometimes striking difficulties experienced by PD patients in attempting to roll over in bed (Lakke, 1985).

The pathophysiology of disturbed proprioception in PD remains unknown. The results of anatomical studies indicate that the basal ganglia receive abundant afferent information. This renders them suitable to serve as a 'comparator' that reweighs the various sensorimotor loops, matches this sensory information to the corollary discharge information and translates afferent information from sensory and motor sources into appropriate motor programs. In PD patients, this afferent information itself is presumably normal, but proprioceptive signals are perhaps abnormally processed within the basal ganglia due to defective higher level integration. This hypothesis is supported by the finding of reduced sensory-evoked brain activations in cortical and subcortical areas in PD patients using positron emission tomography 
(Boecker et al., 1999).

Further supportive evidence for impaired central proprioceptive integration in PD stems from animal work. For example, the pallidal neurons of MPTP monkeys show a greatly enhanced responsiveness to natural proprioceptive stimulation, with decreased directional dependency (Filion et al., 1988). Hemiparkinsonian MPTP monkeys show extinction, contralateral to the lesion, when confronted with simultaneous bilateral stimuli (Schneider et al., 1992). Moreover, the ability to use sensory information may depend on the degree of dopamine deficits in the SN (Jaspers et al., 1989; Martens et al., 1996). Mild deficits affect only the ability to use static proprioceptive stimuli in motor control, whereas more pronounced deficits also affect the ability to overcome the proprioceptive deficit using visual information.

\section{CONCLUSIONS}

Observations on patients and animals with focal lesions of the basal ganglia underscore the significance of the basal ganglia and their projections in regulating normal postural control. A particularly important contribution includes flexibility and the role in gain control of balancecorrecting responses, which permits subjects to change their strategy or individual postural responses, according to the actual needs of the task at hand. Apart from such motor functions, the basal ganglia also seem involved in the cognitive aspects of postural control, including the handling of uncertainty and the ability to lend priority to the most vital elements of a complex postural task. In addition, the involvement of the basal ganglia in processing afferent information is increasingly recognized as another important function with relevance for postural control, possibly via the organization of a body scheme or through online modification of ballistic movements.

\section{ACKNOWLEDGEMENTS}

Jasper E. Visser and Bastiaan R. Bloem were supported by the Prinses Beatrix Fonds.

\section{REFERENCES}

Adamovich SV, Berkinblit MB, Hening W, Sage J, Poizner H. 2001. The interaction of visual and proprioceptive inputs in pointing to actual and remembered targets in Parkinson's disease. Neuroscience 104, 1027-1041.

Albin RL, Young AB, Penney JB. 1995. The functional anatomy of disorders of the basal ganglia. Trends Neurosci 18: 63-64.

Alexander GE, Crutcher MD. 1990. Functional architecture of basal ganglia circuits: neural substrates of parallel processing. Trends Neurosci 13: 266-271.

Allum JH, Keshner EA, Honegger F, Wuetrich R. 1988. Disturbance of posture in patients with Parkinson's disease. In: Amblard B, Berthoz A, Clarac F, eds, Posture and Gait: Development, Adaptation and Modulation. Amsterdam, the Netherlands: Elsevier; 245-257.

Bakker M, Esselink RA, Munneke M, LimousinDowsey P, Speelman JD, Bloem BR. 2004. Effects of stereotactic neurosurgery on postural instability and gait in Parkinson's disease. Mov Disord 19: 1092-1099.

Beckley DJ, Bloem BR, Remler MP. 1993. Impaired scaling of long latency postural reflexes in patients with Parkinson's disease. Electroenceph Clin Neurophysiol 89: 22-28.

Beckley DJ, Bloem BR, Remler MP, Roos RA, van Dijk JG. 1991. Long latency postural responses are modified by cognitive set. Electroenceph Clin Neurophysiol 81: 353-358.

Bejjani BP, Gervais D, Arnulf I, Papadopoulos S, Demeret S, Bonnet AM, Cornu P, Damier P, Agid Y. 2000. Axial parkinsonian symptoms can be improved: the role of levodopa and bilateral subthalamic stimulation. J Neurol Neurosurg Psychiatry 68: 595-600.

Bhatia KP, Marsden CD. 1994. The behavioural and motor consequences of focal lesions of the basal ganglia in man. Brain 117: 859-876. 
Bloem BR, Beckley DJ, Remler MP, Roos RA, van Dijk JG. 1995. Postural reflexes in Parkinson's disease during 'resist' and 'yield' tasks. J Neurol Sci 129, 109-119.

Bloem BR, Beckley DJ, van Dijk JG, Zwinderman AH, Remler MP, Roos RA. 1996. Influence of dopaminergic medication on automatic postural responses and balance impairment in Parkinson's disease. Mov Disord 11, 509-521.

Bloem BR, Bhatia KP. 2004. Gait and balance in basal ganglia disorders. In: Bronstein $\mathrm{A}$, Brandt $\mathrm{T}$, Woollacott MH, Nutt JG, eds, Clinical Disorders of Balance, Posture and Gait, London, England: Arnold; 173-206.

Bloem BR, Grimbergen YA, Cramer M, Valkenburg VV. 2000. "Stops walking when talking" does not predict falls in Parkinson's disease. Ann Neurol 48: 268. [letter]

Bloem BR, Hausdorff JM, Visser JE, Giladi N. 2004a. Falls and freezing in Parkinson's disease: a review of two interconnected, episodic phenomena. Mov Disord 19: 871-884.

Bloem BR, Munneke M, Mazibrada G, Schrag A, Viswanathan $R$, Lees AJ, et al. 2004b. The nature of falling in progressive supranuclear palsy. Mov Disord 19, 359-360. [extended abstract]

Bloem BR, Roos RA. 1995. Neurotoxicity of designer drugs and related compounds. In: De Wollf FA, ed, Handbook of Clinical Neurology, Vol. 21: Intoxications of the Nervous System, Part II. Amsterdam, the Netherlands: Elsevier; 363-414.

Bloem BR, Valkenburg VV, Slabbekoorn M, Willemsen MD. 2001a. The Multiple Tasks Test. Strategies in Parkinson's disease. Exp Brain Res 137: 478-486.

Bloem BR, Valkenburg VV, Slabbekoorn M, Willemsen MD. 2001b. The Multiple Tasks Test: development and normal strategies. Gait Posture 14: 191-202.

Bloem BR, van Vugt JP, Beckley DJ. 2001c. Postural instability and falls in Parkinson's disease. Adv Neurol 87, 209-223.

Bloem BR, Visser JE, Allum JH. 2003. Posturography. In: Hallett, $M$, ed, Handbook of Clinical Neurophysiology. Amsterdam, the Netherlands: Elsevier Science BV; 295-336.

Bloem BR, Visser JE, Carpenter MG. 2001d. Compensatory postural strategies in patients with longstanding balance impairment. In: Burg JCE van der, Fong BF, Hijl MIJ, Huys R, Pijnappels M, Post $A A$, eds, Balance at All Times. Utrecht, the Netherlands: Digital Printing Partners BV; 51-73.
Boecker H, Ceballos-Baumann A, Bartenstein P, Weindl A, Siebner HR, Fassbender T, et al. 1999. Sensory processing in Parkinson's and Huntington's disease: investigations with 3D $\mathrm{H}(2)(15) \mathrm{O}-\mathrm{PET}$. Brain 122: 1651-1665.

Brown LL, Schneider JS, Lidsky TI. 1997. Sensory and cognitive functions of the basal ganglia. Curr Opin Neurobiol 7: 157-163.

Carpenter MG, Allum JH, Honegger F, Adkin AL, Bloem BR. 2004. Postural abnormalities to multidirectional stance perturbations in Parkinson's disease. J Neurol Neurosurg Psychiatry 75: 1245-1254.

Chesselet MF, Delfs JM. 1996. Basal ganglia and movement disorders: an update. Trends Neurosci 19; 417-422.

Dietz V, Berger W, Horstmann GA. 1988. Posture in Parkinson's disease: impairment of reflexes and programming. Ann Neurol 24: 660-669.

Dietz V, Zijlstra W, Prokop T, Berger W. 1995. Leg muscle activation during gait in Parkinson's disease: adaptation and interlimb coordination. Electroenceph Clin Neurophysiol 97: 408-415.

Dimitrova D, Horak FB, Nutt JG. 2004. Postural muscle responses to multidirectional translations in patients with Parkinson's disease. J Neurophysiol 91, 489501.

Filion M, Tremblay L, Bedard PJ. 1988. Abnormal influences of passive limb movement on the activity of globus pallidus neurons in parkinsonian monkeys. Brain Res 444: 165-176.

Hamani C, Saint-Cyr JA, Fraser J, Kaplitt M, Lozano AM. 2004. The subthalamic nucleus in the context of movement disorders. Brain 127: 4-20.

Hirsch EC, Graybiel AM, Duyckaerts C, Javoy-Agid F. 1987. Neuronal loss in the pedunculopontine tegmental nucleus in Parkinson disease and in progressive supranuclear palsy. Proc Natl Acad Sci USA 84: 5976-5980.

Horak FB, Frank J, Nutt J. 1996. Effects of dopamine on postural control in parkinsonian subjects: scaling, set, and tone. J Neurophysiol 75, 2380-2396.

Horak FB, Nutt JG, Nashner LM. 1992. Postural inflexibility in parkinsonian subjects. J Neurol Sci 111: 46-58.

Jaspers RM, Berkelbach van der Sprenkel JW, Cools AR. 1989. Progressive pathology of the caudate nucleus, the substantia nigra pars reticulata and the deeper layers of the colliculus superior: acute behavioural and metabolic effects of intrastriatal kainic acid. Neuroscience 28: 159-169. 
Karnath, H.O., Ferber, S., and Dichgans, J. 2000. The neural representation of postural control in humans. Proc Natl Acad Sci USA 97, 13931-13936.

Keijsers NL, Admiraal MA, Cools AR, Bloem BR, Gielen CC. 2005. The accuracy of pointing movements to remembered visual targets in Parkinson's disease. Eur J Neurosci 21: 239-248.

Kitamura, J., Nakagawa, H., linuma, K., Kobayashi, M., Okauchi, A., Oonaka, K., et al. 1993. Visual influence on center of contact pressure in advanced Parkinson's disease. Arch Phys Med Rehabil 74: 1107-1112.

Klockgether T, Borutta M, Rapp H, Spieker S, Dichgans J. 1995. A defect of kinesthesia in Parkinson's disease. Mov Disord 10: 460-465.

Krack P, Batir A, Van Blercom N, Chabardes S, Fraix V, Ardouin C, et al. 2003. Five-year follow-up of bilateral stimulation of the subthalamic nucleus in advanced Parkinson's disease. N Engl J Med 349: 1925-1934.

Labadie EL, Awerbuch GI, Hamilton RH, Rapesak SZ. 1989. Falling and postural deficits due to acute unilateral basal ganglia lesions. Arch Neurol 46: 492-496.

Lakke JP. 1985. Axial apraxia in Parkinson's disease. J Neurol Sci 69: 37-46.

Lee MS, Rinne JO, Marsden CD. 2000. The pedunculopontine nucleus: its role in the genesis of movement disorders. Yonsei Med J 41: 167-184.

Limousin P, Krack P, Pollak P, Benazzouz A, Ardouin C, Hoffmann D, Benabid AL. 1998. Electrical stimulation of the subthalamic nucleus in advanced Parkinson's disease. N Engl J Med 339: 1105-1 111.

Lundin-Olsson L, Nyberg L, Gustafson Y. 1997. "Stops walking when talking" as a predictor of falls in elderly people. Lancet $349: 617$. [Letter]

Marsden CD. 1982. The mysterious motor function of the basal ganglia: the Robert Wartenberg Lecture. Neurology 32: 514-539.

Martens DJ, Whishaw IQ, Miklyaeva EI, Pellis SM. 1996. Spatio-temporal impairments in limb and body movements during righting in an hemiparkinsonian rat analogue: relevance to axial apraxia in humans. Brain Res 733: 253-262.

Masdeu JC, Alampur U, Cavaliere R, Tavoulareas G. 1994. Astasia and gait failure with damage of the pontomesencephalic locomotor region. Ann Neurol 35: 619-621.

Maurer C, Mergner T, Xie J, Faist M, Pollak P, Lucking $\mathrm{CH}$. 2003. Effect of chronic bilateral subthalamic nucleus STN) stimulation on postural control in Parkinson's disease. Brain 126: 1146-1163.

Moore, G., Robertson, D., Kuasar, S.A., and Taylar, G. 2000. Postural awareness and falls in Parkinson's disease. Proceedings of a conference on Multidisciplinary Care in Parkinson's Disease and Parkinsonism, from Science to Practice. London, UK; 14.

Munro-Davies LE, Winter J, Aziz TZ, Stein JF. Munro1999. The role of the pedunculopontine region in basal-ganglia mechanisms of akinesia. Exp Brain Res 129: 511-517.

Nandi D, Aziz TZ, Giladi N, Winter J, Stein JF. 2002a. Reversal of akinesia in experimental parkinsonism by GABA antagonist microinjections in the pedunculopontine nucleus. Brain 125: 2418-2430.

Nandi D, Liu X, Winter JL, Aziz TZ, Stein JF. 2002 b. Deep brain stimulation of the pedunculopontine region in the normal non-human primate. $\mathrm{J}$ Clin Neurosci 9: 170-174.

Pahapill PA, Lozano AM. 2000. The pedunculopontine nucleus and Parkinson's disease. Brain 123: $1767-$ 1783.

Parent A, Cicchetti F. 1998. The current model of basal ganglia organization under scrutiny. Mov Disord. 13: 199-202.

Parent A, Hazrati LN. 1995a. Functional anatomy of the basal ganglia. I. The cortico-basal ganglia- thalamocortical loop. Brain Res Brain Res Rev 20: 91-127.

Parent A, Hazrati LN. 1995b. Functional anatomy of the basal ganglia. II. The place of subthalamic nucleus and external pallidum in basal ganglia circuitry. Brain Res Brain Res Rev 20: 128-154.

Samii A, Nutt JG, Ransom BR. 2004. Parkinson's disease. Lancet 363: 1783-1793.

Schieppati M, Nardone A. 1991. Free and supported stance in Parkinson's disease. Brain 114: 1227-1244.

Schneider JS, McLaughlin WW, Roeltgen DP. 1992. Motor and nonmotor behavioral deficits in monkeys made hemiparkinsonian by intracarotid MPTP infusion. Neurology 42: 1565-1572.

Snow BJ, Vingerhoets FJ, Langston JW, Tetrud JW, Sossi V, Calne DB. 2000. Pattern of dopaminergic loss in the striatum of humans with MPTP induced parkinsonism. J Neurol Neurosurg Psychiatry 68, 313-316.

Speelman JD. 1991. Parkinson's disease and stereotaxic neurosurgery. Amsterdam, the Netherlands: Thesis.

Su PC, Tseng HM, Liou HH. 2002. Postural asymmetries following unilateral subthalomotomy for advanced 
Parkinson's disease. Mov Disord 17: 191-194.

Takakusaki K, Oohinata-Sugimoto J, Saitoh K, Habaguchi

T. 2004. Role of basal ganglia-brainstem systems in the control of postural muscle tone and locomotion.

Prog Brain Res 143: 231-237.
Deep-Brain Stimulation for Parkinson's Disease Study Group. 2001. Deep-brain stimulation of the subthalamic nucleus or the pars interna of the globus pallidus in Parkinson's disease. N Engl J Med 345: 956-963. 

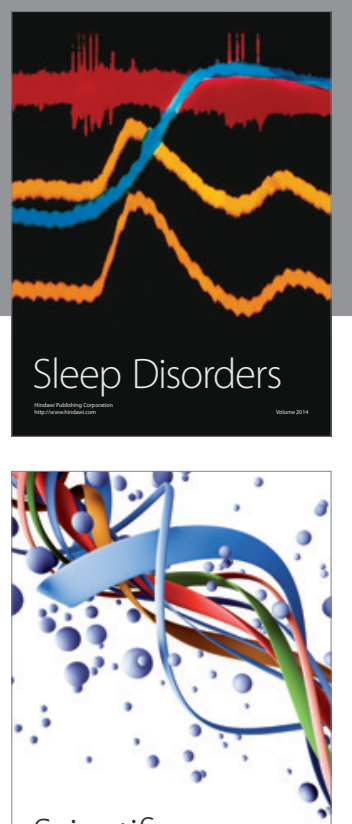

Scientifica
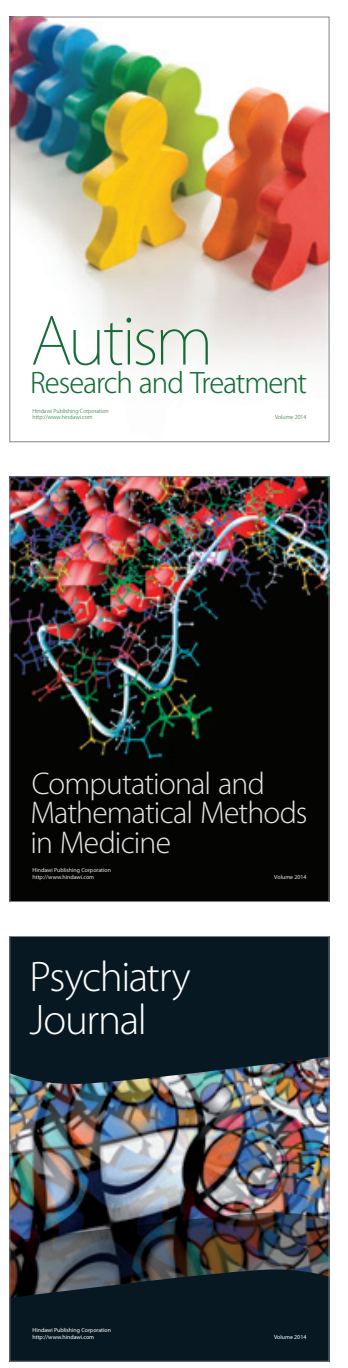
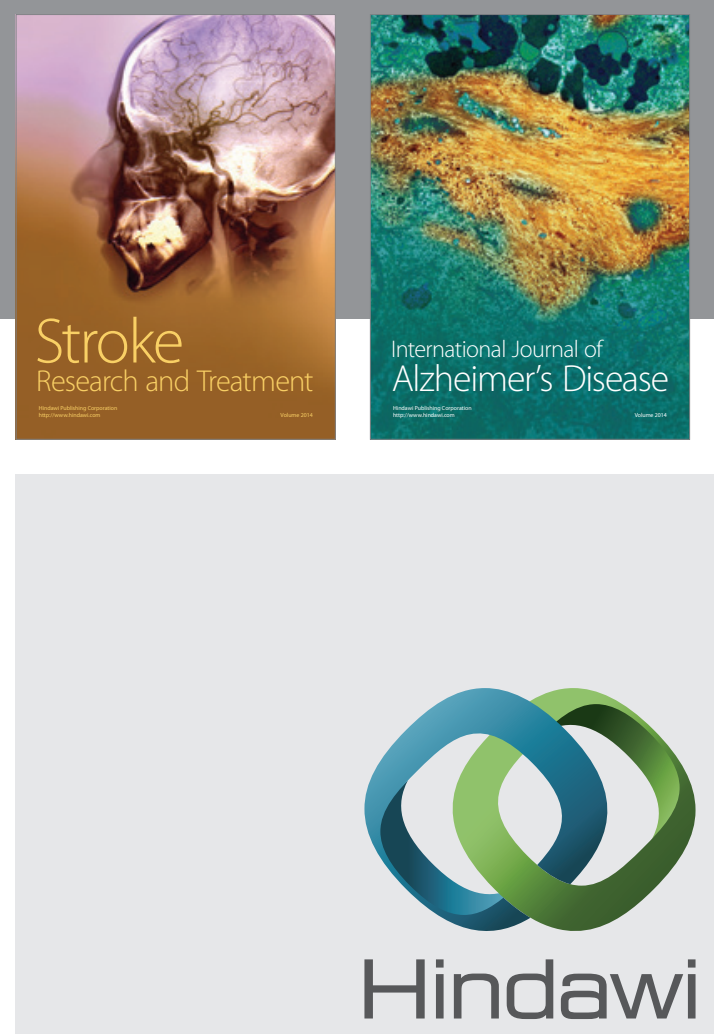

Submit your manuscripts at

http://www.hindawi.com
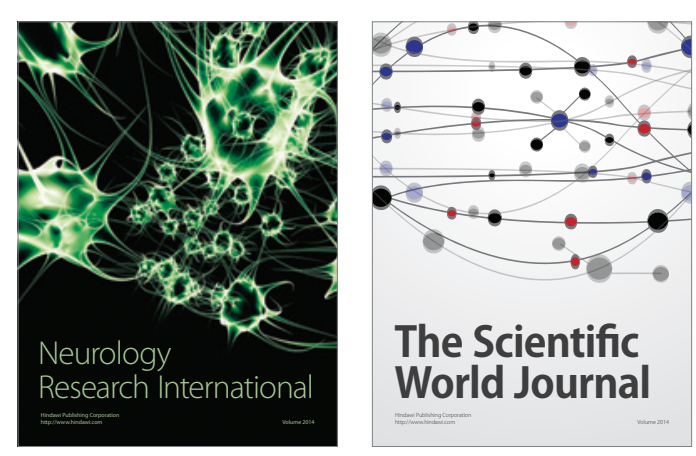

The Scientific World Journal

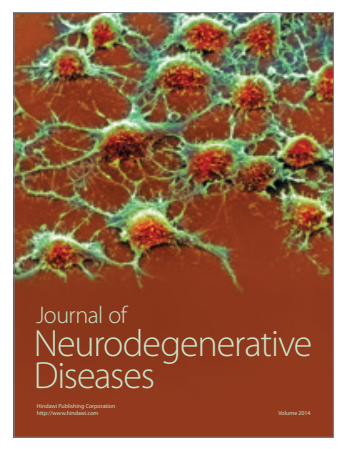

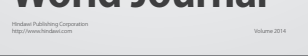

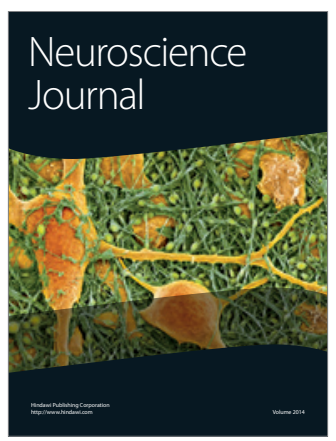

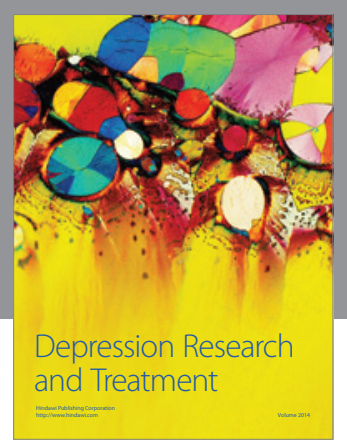
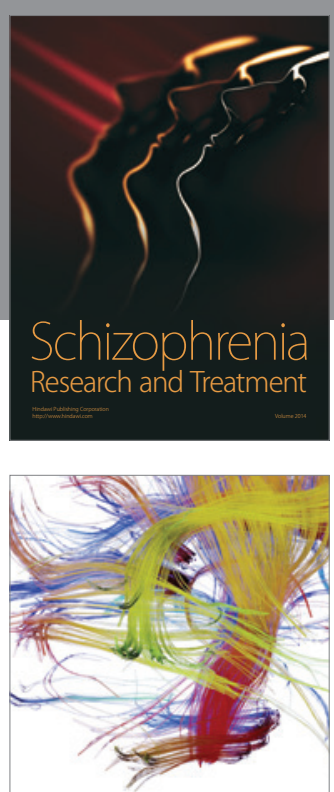

Brain Science

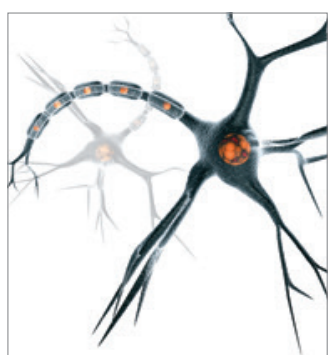

Neural Plasticity
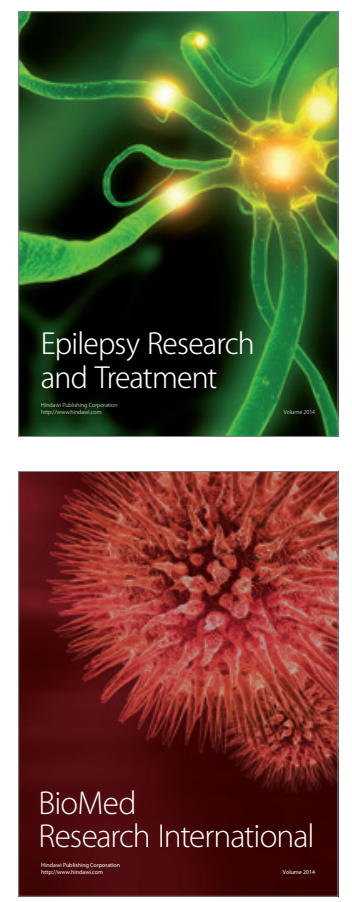

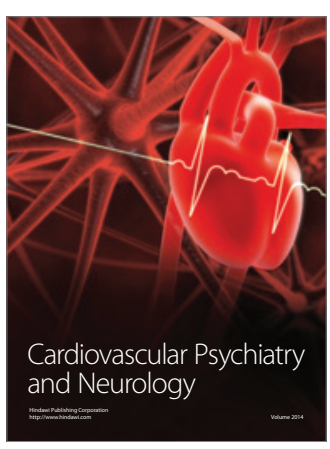

Parkinson's

Disease
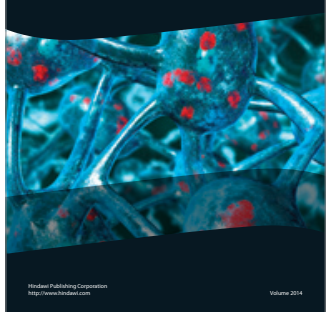